\title{
PRIMER IMPACTO: PROGRAMA DE DETECCIÓN DE DISTRÉS Y ATEN- CIÓN PSICOSOCIAL PARA PACIENTE RECIÉN DIAGNOSTICADO Y SUS FAMILIARES
}

\author{
FIRST IMPACT: SCREENING PROGRAM FOR DISTRESS AND PSYCHOSOCIAL CARE \\ FOR NEWLY DIAGNOSED PATIENTS AND THEIR FAMILIES
}

Belén Fernández, Eva Bejar, María del Mar Campos

Asociación Española Contra el Cáncer

Resumen

En 1997, la Red Oncológica Nacional (National Comprehensive Cancer Network, NCCN) creó una comisión multidisciplinar para examinar cómo integrar los cuidados psicosociales de los enfermos oncológicos en la valoración de rutina y observó que el concepto de distrés, entendido como sufrimiento, era el mejor término genérico para representar la variedad de preocupaciones que experimentan los pacientes y no conllevaba el estigma de otras palabras usadas para los síntomas emocionales. El presente trabajo expone la implantación del Programa Primer Impacto, un proceso de asistencia psicosocial que pretende abordar las necesidades bio-psico-sociales del enfermo recién diagnosticado de cáncer y sus familiares de manera integral e inmediata. La utilización un método sencillo de screnning de distrés (entrevista semiestructurada y termómetro de distrés) nos permite realizar un triage de necesidades, decidir las prioridades en la asistencia y realizar las primeras medidas de atención psicosocial. Revisamos la bibliografía actual en esta disciplina, en base a una exhaustiva revisión de la literatura y aportamos nuestra propia experiencia en la implantación de un modelo de detección de distrés estructurado, en cuanto a los recursos necesarios para una adecuada implantación, así como
Abstract

The National Comprehensive Cancer Network created in 1997 a multidisciplinar comission whit the aim to examine how integrate the psychosocial attention of cancer patient into the routine assessment of this patients. This group noted that distress, treated as emotional suffer was the best construct to show the variety of concerns experimented by the cancer patients free from the stigma of others concepts used to define emotional symptoms. This paper show the develop of a program called First Impact (Primer Impacto), consisting a psychosocial attention program which intends to respond immediately to all bio-psycho-social needs of newly diagnosed patient and their relatives. The assessment by a simple screening tool (semi-structured interview and the Distress Thermometer) lets us to give the first psychosocial cares. An exhaustive review of the available literature, our experience to present the necessary resources to an adequate implantation of a structured detection of distress protocol, and the results obtained from its implementation in different hospitals and the Spanish Association Against Cancer centers are provided.

Keywords: Distress, cancer, screening, triage, psychosocial adjustment.

\section{Correspondencia:}


los resultados obtenidos desde su puesta en marcha en diferentes hospitales y sedes de la Asociación Española Contra el Cáncer.

Palabras clave: Distrés, malestar emocional, cáncer, triage, adaptación psicológica.

\section{INTRODUCCIÓN}

El diagnóstico de cáncer está asociado a una amenaza para la salud con repercusiones en todas las esferas de la vida de la persona enferma y de sus familiares: a nivel social, laboral, económico y familiar. Este proceso de ajuste a la nueva situación puede producirse de manera adecuada sin que en el paciente y su familia aparezcan problemas susceptibles de intervención. Sin embargo, la experiencia de enfermedad conlleva, muy frecuentemente, la aparición de sentimientos dolorosos que pueden derivar en altos niveles de sufrimiento y en problemas de adaptación a la enfermedad $^{(1)}$.

Diversos estudios sugieren que entre el $25-50 \%$ de la población oncológica presenta alteraciones psicológicas derivadas del proceso de enfermedad ${ }^{(2,-4)}$. Con respecto a la morbilidad psicológica de los pacientes con cáncer en España, un estudio multicéntrico desarrollado de forma conjunta por el Instituto Catalán de Oncología y dos centros de Italia y Portugal muestran que el $28,5 \%$ de los pacientes presentan síntomas ansioso-depresivos que precisan derivación a salud mental ${ }^{(5)}$. En esta misma línea, un estudio más reciente desarrollado por Gil et al., para determinar la prevalencia de trastornos mentales, en una amplia muestra de pacientes españoles con cáncer, señala que el $24 \%$ de los mismos presenta criterios para un diagnóstico psicopatológico según el DSM-IV. De ellos, el $77 \%$ corresponde a trastornos adaptativos ${ }^{(6)}$. Los datos, anteriormente expuestos, señalan que el diagnóstico de cáncer se convierte en un momento de crisis vital de la persona que implica una importante transformación así como un reajuste de comportamientos, actitudes, emociones y decisiones.

Las emociones desempeñan un papel fundamental en la adaptación a la enfermedad, pudiendo ser factores de riesgo o de protección. El soporte emocional debería, por tanto, iniciarse en el primer momento del diagnóstico, trabajando las emociones que tal noticia conlleva, y de esta manera poder prevenir un mal manejo de las mismas que suponga, por tanto, un proceso desadaptativo de la enfermedad $^{(7)}$. En este contexto, un concepto de gran relevancia e interés para los diferentes grupos de investigación ha sido determinar hasta dónde una intervención inmediata e integral puede ofrecer resultados positivos en cuanto a minimizar el posible impacto emocional en los pacientes de cáncer y sus familiares. Ya en 1991, Iscoe et al., relatan que la evaluación del distrés en el paciente poco después de recibir el diagnóstico de cáncer es el mejor predictor en cuanto a la posibilidad de desarrollar problemas de ajuste posterior ${ }^{(8)}$. Vargas et al., aportan que cuanto más rápido se vea el enfermo involucrado en un proceso de apoyo integral, mejor será su capacidad de adaptación, de ahí la importancia de priorizar la intervención en pacientes recién diagnosticados ${ }^{(9)}$. En esta misma línea, Fallowfield et al., destacan que muchos de los problemas psicosociales que aparecen en pacientes podrían ser evitados y/o minimizados a través de unas adecuadas habilidades de comunicación por parte de los clínicos. En un estudio con mujeres con cáncer de mama, se observa que 
aquellas que estaban satisfechas con la comunicación transmitida por su cirujano en cuanto a su diagnóstico y las posibilidades terapéuticas, mostraron menor morbilidad psiquiátrica durante los tres años siguientes a la finalización del tratamiento ${ }^{(10)}$. Los trabajos de Kiss et al., Ilegan a conclusiones similares, destacando que los oncólogos pueden jugar un papel importante no sólo en identificar distrés psicosocial sino también en proporcionar una adecuada información y apoyo básico a los pacientes y sus familiares. Además, pueden ofrecer derivación a otros servicios de apoyo psicosocial especializado a aquellos pacientes que presenten un nivel de distrés más elevado o un escaso apoyo por parte de su red social. Por este motivo, los autores concluyen que el grado de distrés así como el grado de apoyo social percibido por el paciente debería ser evaluado lo antes posible ${ }^{(11)}$.

En 1997, la Red Oncológica Nacional de Estados Unidos (National Comprehensive Cancer Network, NCCN) creó una comisión multidisciplinar para examinar cómo integrar los cuidados psicosociales en la valoración médica de rutina. Se preguntaba a los pacientes acerca de sus preocupaciones psicosociales, y la comisión de la NCCN observó que el distrés, entendido como "Una experiencia emocional desagradable de naturaleza psicológica, social y/o espiritual que se extiende en un continuo de sentimientos normales de vulnerabilidad, tristeza y miedos a problemas más graves de depresión, ansiedad, pánico, aislamiento social y crisis espiritual o de creencias", era el mejor término para representar la variedad de preocupaciones emocionales que experimentan los pacientes con cáncer ${ }^{(12)}$. El distrés no es un término clínico preciso que aparezca en el DSM-IV como diagnóstico psiquiátrico, pero se constituye como un criterio clínico significativo para clasificar varios de los trastornos del estado de ánimo. Una de las razones para su adopción en el ámbito de la oncología es que el término distrés a menudo es más útil que términos psiquiátricos como la ansiedad o la depresión Es más fácil de entender por los profanos y no conlleva el estigma asociado a etiquetas como psiquiátrico, psicosocial y problemas emocionales. Por lo general, es bien comprendido por los profesionales sanitarios no especializados en salud mental y facilita la evaluación rápida a través de autoinforme del paciente ${ }^{(13)}$.

Söllner et al., ponen de manifiesto una necesidad real de elaboración de métodos que ayuden a identificar los posibles problemas sociales y nivel de distrés que tienen los pacientes y familiares para, de esta forma, ofrecerles los recursos necesarios que puedan cubrir dichas necesidades. Entre estos métodos, los autores destacan: a) utilización de métodos de screening, y b) proporcionar un adecuado entrenamiento a las personas encargadas de detectar las diferentes demandas y necesidades de los pacientes ${ }^{(14)}$. Sin embargo, aún no se ha establecido un modelo operativo en el que los diferentes servicios asistenciales se puedan enlazar entre sí y adecuar a las necesidades que presentan los pacientes de cáncer y al grado de severidad de su sintomatología. Diversas guías de práctica clínica, publicadas en Australia, Canadá y EEUU, recogen un conjunto de recomendaciones elaboradas en base a las evidencias científicas disponibles, para ayudar a los clínicos y a los pacientes en la toma de decisiones sobre los cuidados psicosociales más adecuados en oncología. En definitiva, definen la estructura y la dirección de las intervenciones psicosociales en pacientes con cáncer y sus familiares. En todas ellas aparece como noción emergente de gran relevancia el concepto de triage, el cual hace referencia a un método de selección y clasificación de pacientes, basado en sus necesidades terapéuticas y los recursos disponibles para su atención. Como 
señala Mitchel, cada persona reacciona de forma diferente y con distinta intensidad emocional ante una misma situación estresante. Por este motivo, es necesario desarrollar un proceso de selección previa que permita proporcionar la intervención psicosocial más adecuada a las necesidades de la persona y en el momento más adecuado $^{(15)}$. Hutchison y colaboradores proponen un modelo gradual de intervención psicosocial, donde se definen los pasos y la toma de decisiones para ajustar las estrategias terapéuticas a los niveles y naturaleza del malestar emocional del paciente. El modelo gradual de intervención psicosocial se basa en el concepto de ajuste de tratamiento como una extensión de los modelos de screening. La rutina del screening aplicada a la intervención psicosocial resulta efectiva a la hora de superar las dificultades existentes en la detección de los pacientes que necesitan una intervención y para evaluar el nivel y el tipo de necesidad, de este modo se puede ofrecer la intervención más adecuada a cada caso en particular. La valoración de los pacientes debe ser continua mientras la persona se encuentre en la red de atención, con la posibilidad de enlazar con otro servicio o derivar hacia un nivel anterior una vez que las necesidades psicosociales del paciente hayan cambiado ${ }^{(16)}$.

El distrés es un problema significativo para muchas personas afectadas de cáncer en nuestro país pero existe una amplia red de intervenciones psicosociales disponibles para su asistencia. Es esencial que esta asistencia venga acompañada de un fácil acceso a los servicios ya que éstos pueden ser eficaces, pero sin una amplia difusión, su impacto en la comunidad será limitado. El modelo gradual de intervención es un modelo genérico que puede ser adaptado a cualquier tipo de cáncer y puede representar una base para conceptualizar la estructura y la función de una amplia red de servicios entrelazados entre sí. Para adaptar el modelo a los profesionales se han de identificar los servicios existentes y adecuarlos a los diferentes niveles del modelo. Para ello, es necesario poder acceder a un rango de intervenciones psicosociales que ofrezcan cobertura en diferentes niveles de atención, tales como información, grupos de apoyo mutuo, counselling e intervenciones psiquiátricas o psicosociales. En muchos casos, esos servicios vendrán ofrecidos por diferentes profesionales. La cuestión que se plantea por tanto es cómo enlazar estos servicios en el marco de un modelo integral de apoyo al paciente oncológico y sus familiares ${ }^{(17)}$. En varios centros integrales de cáncer se ha puesto en marcha modelos o protocolos de detección de distrés. A pesar de que existen marcadas diferencias entre ellos, la mayoría de los modelos incluyen tres etapas consecutivas de desarrollo: administración del examen de detección, puntaje y derivación. La mayor parte de los protocolos desarrollados, hasta el momento, han sido enfocados hacia el paciente; sin embargo, ya se están formulando algunos procedimientos de detección de distrés dirigidos a los familiares ${ }^{(18)}$.

En el presente trabajo expondremos la guía de intervención del programa de detección de distrés psicosocial para pacientes recién diagnosticados de cáncer y sus familiares que llevamos realizando desde hace dos años en la Asociación Española Contra el Cáncer (AECC).

\section{ASPECTOS FORMALES DEL PROGRAMA PRIMER IMPACTO}

Primer Impacto es un proceso de asistencia psicosocial que pretende abordar las necesidades bio-psico-sociales del enfermo recién diagnosticado de cáncer y sus familiares de manera integral e inmediata, en la figura 1 se detalla la ficha técnica. En definitiva, es un programa de detección de distrés que se constituye como un modelo 
Figura 1. Ficha técnica.

\begin{tabular}{|c|c|}
\hline Denominación & Primer Impacto \\
\hline Beneficiarios & $\begin{array}{l}\text { - Enfermos mayores de } 18 \text { años con diagnóstico de malignidad } \\
\text { confirmado } \\
\text { - } \quad \text { Tiempo máximo transcurrido desde el diagnóstico } \underline{3 \text { meses }} \\
\text { - En todos los estadios de la enfermedad (excepto recidivas) } \\
\text { - } \quad \underline{\text { Familiares de enfermos que cumplan los requisitos anteriores y padres }} \\
\underline{\text { de niños con diagnóstico de cáncer. }}\end{array}$ \\
\hline Objetivos & $\begin{array}{l}\text { - Identificar precozmente aquellos síntomas (físicos, psicológicos y/o } \\
\text { sociales) que son percibidos como amenaza por el enfermo y su } \\
\text { familia. } \\
\text { - Evaluar el grado de distress que presenta el enfermo y su familia tras } \\
\text { recibir el diagnóstico. } \\
\text { - Derivar de manera efectiva a los pacientes y familiares que demanden } \\
\text { atención médica, psicológica o social al servicio más adecuado y } \\
\text { ajustado a sus necesidades. }\end{array}$ \\
\hline $\begin{array}{l}\text { Lugar de } \\
\text { intervención }\end{array}$ & $\begin{array}{l}\text { - Hospitales } \\
\text { - Sedes aeec. Dos modalidades de atención: } \\
\square \text { Presencial } \\
\square \quad \text { Telefónica }\end{array}$ \\
\hline Recursos & $\begin{array}{l}\text { HUMANOS } \\
\text { - } \quad \text { Responsable Primer impacto (Psicólogo/Trabajador Social/ Médico/ } \\
\text { Due/coordinadores de voluntariado) } \\
\text { MATERIALES } \\
\text { - } \quad \text { Materiales de difusión del programa (cartel y octavilla) }\end{array}$ \\
\hline Intervención & $\begin{array}{l}\text { La intervención tiene una duración de } 1 \text { o } 2 \text { sesiones máximo y se } \\
\text { desarrolla en tres fases: } \\
\text { Fase de ventilación y exploración; establecimiento de una relación de } \\
\text { confianza que facilite el desahogo emocional a través de la expresión } \\
\text { de pensamientos y emociones. } \\
\text { Fase de triage o detección de necesidades; método de evaluación y } \\
\text { screening que facilita una adecuada labor de selección y clasificación } \\
\text { que garantice una rápida derivación a los niveles de atención que el } \\
\text { paciente necesite. } \\
\text { Fase de normalización y educación; normalizar las reacciones que se } \\
\text { están experimentando, explorar la estrategias de afrontamiento que se } \\
\text { han puesto en marcha o bien animar a que se movilicen los recursos } \\
\text { disponibles, ofrecer información acerca de los recursos de ayuda } \\
\text { disponibles en la aecc. }\end{array}$ \\
\hline Evaluación & $\begin{array}{l}\text { Intervención } \\
\text { - } \quad \text { Entrevista semiestructurada } \\
\text { - } \quad \text { Termómetro de distress } \\
\text { Seguimiento } \\
\text { - } \quad \text { Ficha de registro } \\
\text { - } \quad \text { Cuestionario satisfacción }\end{array}$ \\
\hline
\end{tabular}


estructurado de intervención psicosocial gradual que ofrece una adecuada cobertura a las necesidades del enfermo y/o sus familiares en el momento del diagnóstico, permitiendo el cumplimiento óptimo de las siguientes funciones:

1.- Identificar de forma rápida las necesidades psicosociales de los pacientes mediante un sistema estandarizado de clasificación.

2.- Asegurar la priorización en función del nivel de distrés del paciente y/o familiar.

3.- Determinar el servicio o programa más adecuado para cada caso.

4.- Disminuir la posible congestión de los servicios, mejorando el flujo de pacientes dentro de los programas de atención psicosocial de la AECC. De hecho, los tratamientos de atención temprana reducen los gastos generales, simplifican y mejoran el acceso a los servicios adecuados y la atención a las personas que lo necesitan y mejoran la elección del tratamiento.

El modelo aplicado en el protocolo de intervención de Primer Impacto establece cuatro niveles de apoyo o atención:

- Nivel 1 o distrés mínimo: este nivel cubriría la necesidad creciente de información que presentan los enfermos y familiares recién diagnosticados sobre cualquier aspecto relacionado con la enfermedad que puede generar en esta población un distrés mínimo y una necesidad de apoyo general en torno a la enfermedad. Los servicios que pueden ofrecer este tipo de ayuda serían el servicio de ayuda telefóni$\mathrm{ca}$, asesoramiento online y materiales educativos (folletos, guías, etc.). Todos ellos se caracterizan por ser servicios de baja intensidad, no presenciales, de bajo coste $y$ de fácil acceso.

- Nivel 2 o atención específica: en este nivel de atención se incluyen a pacientes y familiares que presentan un nivel de distrés moderado y en los que se detecta una necesidad de asesoramiento o apoyo psico- social de duración breve. Los servicios que pueden ofrecer cobertura a estos pacientes serían grupos psicoeducativos, grupos de apoyo mutuo, atención y/o apoyo profesional telefónico.

- Nivel 3 o atención especializada: dirigido a pacientes y familiares que presentan un nivel severo de distrés que requieren de una intervención especializada en el abordaje de su problemática, así como un seguimiento de su estado psicosocial. Se requieren servicios de alta intensidad ofrecida por psicólogos, psiquiatras, trabajadores sociales $u$ otros profesionales en función de la problemática específica.

- Nivel 4 o atención en crisis: dirigido a pacientes con un nivel de distrés severo y que requerirá de una intervención en crisis o atención para casos complejos y que vendría ofrecida por un equipo multidisciplinar de profesionales de salud mental.

\section{¿CÓMO SE DESARROLLA EL PROGRAMA PRIMER IMPACTO?}

\section{Cuestiones previas para facilitar el adecuado desarrollo del programa}

A partir de nuestra propia experiencia con otro tipo de intervenciones que se realizan en el ámbito hospitalario y a las recomendaciones realizadas por otros autores, sabíamos que un componente esencial para optimizar los resultados finales era preparar exhaustivamente el terreno. Por lo tanto, resultaba particularmente importante, antes de tratar de introducir el programa, lograr el apoyo de los administradores y gerentes de hospitales. Así, fue imprescindible una exhaustiva labor de promoción y gestión en los centros hospitalarios. Con este objeto se desarroIlaron distintas acciones que se detallan a continuación:

- Reuniones de presentación del programa con el gerente y director médico del hospital de referencia. Con el objetivo de 
establecer una coordinación estructurada con los distintos niveles asistenciales.

- Revisión o firma de convenio de colaboración para el desarrollo del programa.

- Campaña informativa en cada hospital, en función de su gestión y estructura. Se desarrolla una variedad de acciones informativas para profesionales sanitarios con el objeto de crear un estado de opinión favorable que permita optimizar la participación de pacientes y familiares en el programa.

A nivel de organización, inicialmente y previo a la realización del protocolo de intervención, también es importante asegurarse de que se dispone de una variedad de servicios de apoyo para los pacientes y/o familiares que presenten necesidades y niveles de distrés significativos. Sin duda, esta diversidad de servicios es condición necesaria para asegurar la continuidad de la atención de seguimiento y el tratamiento apropiado. No menos importante es definir un protocolo de triage con pautas y algoritmos claramente establecidos que puedan ser aplicados por los diferentes profesionales que lo desarrollarán en la práctica diaria, pero no exento de cierta flexibilidad clínica que permita que algunas decisiones se tomen en base al juicio del profesional para anular la aparición de posibles falsos negativos y falsos positivos.

El funcionamiento eficaz del modelo necesita de la existencia de un equipo de profesionales que pueda identificar las necesidades de los pacientes, decidir las prioridades y realizar las primeras medidas de apoyo psicosocial (información y desahogo emocional). Para ello, dichos profesionales deben estar cualificados y formados en la aplicación del modelo así como en las herramientas de triage utilizadas. Ha de conocer perfectamente los instrumentos de evaluación a aplicar, realizar una rápida evaluación, recogiendo los datos expresados por el paciente y dirigiendo la entrevista cuando sea necesario, para objetivar al máximo el distrés y las necesidades psicosociales del paciente.

\section{Captación de beneficiarios y desarrollo del protocolo de intervención}

El proceso se inicia cuando el paciente entra en contacto con el programa a través de las diferentes vías de entrada (hospitales, sedes y servicio de atención telefónica Infocáncer de la AECC) y finaliza en el momento en que el paciente es derivado al servicio y profesional que su situación inicial requiera. Así, coincidiendo con la visita al centro hospitalario correspondiente o a la sede de la AECC se invita a participar en el programa, a todos aquellos sujetos que cumplan los criterios de inclusión, explicándole los objetivos del mismo.

La intervención de Primer Impacto se realiza en una o dos sesiones cuyo objetivo fundamental es restablecer en el paciente o en el familiar su capacidad de afrontamiento inmediato a través del suministro de un apoyo adecuado. Para ello es necesario aliviar de forma inmediata los síntomas agudos de estrés y facilitar la disposición de recursos de ayuda que le permitan recuperar un adecuado nivel de funcionamiento. La intervención se desarrolla en tres fases diferentes: ventilación, normalización y triage.

\section{Fase de ventilación y exploración}

El primer paso necesario es el establecimiento de una relación de confianza que facilite el desahogo emocional a través de la expresión de pensamientos y emociones. Invitar al paciente a hablar, escuchar los hechos y sentimientos de manera cuidadosa, sintetizar/reflejar los mismos, efectuar declaraciones empáticas, transmitir aceptación e interés por comunicarse. Todo ello, para que el paciente se sienta comprendido al mismo tiempo que se reduce la intensidad del aturdimiento 
emocional y se inicia la reactivación de las capacidades de resolución de problemas

\section{Fase de normalización}

Los pasos en esta fase se concretan en: resumir los aspectos más significativos, normalizar las reacciones que está experimentando, explorar las estrategias de afrontamiento que han puesto en marcha o bien animar a que movilicen los recursos que tienen.

\section{Fase de triage o detección de distrés}

El equipo de profesionales que desarroIla el programa (médicos, psicólogos, trabajadores sociales y coordinadores de voluntariado) es responsable de la acogida y evaluación de las necesidades y distrés del paciente y/o familiar y de realizar los trámites de derivación cuando sea pertinente. Los instrumentos que se utilizan para realizar la evaluación son el Termómetro de distrés (DT) $(19,20,21,22,23,24,25,26)$, escala visual analógica de 10 puntos, donde el anclaje inferior es el punto cero "ausencia de distrés" y el superior el punto 10 "distrés extremo" y una entrevista semiestructurada que permita conocer las necesidades que están generando el sufrimiento del paciente. En función de los puntajes obtenidos en la evaluación se procede a la derivación o no del usuario en función del nivel de necesidad y la urgencia con la que debe ser atendido. En la figura 2 se detalla el algoritmo de intervención.

\section{MÉTODO}

\section{Participantes}

Entre enero de 2011 y marzo de 2012, los 1.525 participantes en el programa de detección fueron reclutados en 25 sedes de la aecc y 27 hospitales públicos y privados de 25 provincias. Los criterios de inclusión en el programa fueron:

- Enfermos mayores de 18 años.

- Diagnóstico de malignidad confirma-

Figura 2. Algoritmo de intervención Primer Impacto.

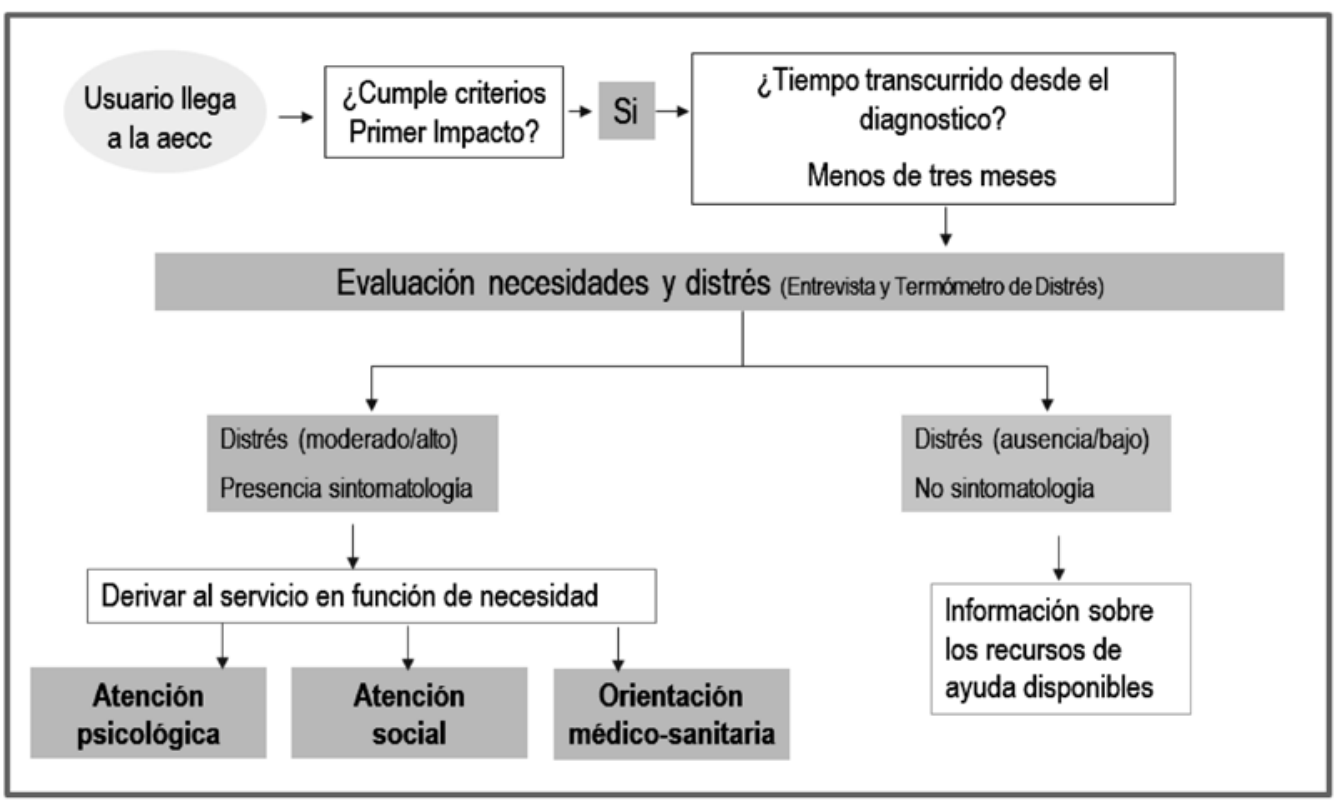


do por anatomía patológica en cualquier localización.

- Todos los estadios (excepto recidiva).

- Tiempo máximo transcurrido desde el diagnóstico de 3 meses.

- Familiares de cualquier rango de enfermos que cumplan los requisitos anteriores.

- Padres de niños con diagnostico de cáncer infantil.

\section{Instrumentos}

Autoinforme. El Termómetro de distrés (DT). Las directrices de la NCCN proporcionan un algoritmo para identificar de forma rápida a los pacientes con un grado de malestar emocional importante. Un resultado de 4 o más (en valoración inicial), Ilevaría al profesional a realizar más preguntas para determinar la causa del sufrimiento y derivar al paciente al servicio psicosocial más adecuado. En definitiva, se requiere de ayuda adicional para identificar la presencia de problemas referidos al bienestar físico, práctico, emocional, familiar o social que puedan contribuir a generar dicho malestar emocional. Con este objeto, el Termómetro de distrés se acompaña de una entrevista.

Entrevista. Se ha diseñado una entrevista semiestructurada "ad hoc" en función de los factores moduladores que han sido estudiados y han resultado relevantes en la bibliografía, la consulta a expertos y la interacción con pacientes oncológicos. En la misma se trata de identificar la naturaleza y fuente de distrés en tres ámbitos, físico, psicológico y social. Concretamente, se incluyen datos sociodemográficos (edad, sexo, estado civil, nivel de la educación y la situación laboral), aspectos relacionados con el diagnóstico de la enfermedad, información recibida sobre tratamientos, efectos secundarios, pronóstico, evolución, etc. Problemas emocionales derivados del diagnóstico, estrategias de afrontamiento de la experiencia, modalidad de comunicación familiar. Finalmente, en el ámbito de necesidades sociales se contemplan problemas laborales, económicos y aquellos relacionados con la familia y la red social disponible.

\section{Análisis estadístico}

Para la descripción de las características de los participantes se ha utilizado el cálculo de las frecuencias absolutas y relativas para las variables nominales, mientras que para las variables continuas se empleó el cálculo de un valor central, media o mediana. La búsqueda de factores de riesgo se realizó mediante análisis de regresión logística binaria, tanto en el análisis univariado como en el multivariado. En el análisis estadístico bivariante se utilizó Chi-cuadrado y la elección de variables en el multivariado se efectuó por el método hacia adelante de Wald. Estos análisis se acompañan de la odds ratio y el correspondiente intervalo de confianza del 95\%. Los datos fueron procesados por el paquete estadístico SPSS (Stadistical Package for Social Sciences, versión 19.0) para windows.

\section{RESULTADOS}

\section{Características demográficas y clínicas}

Los datos referentes a las variables sociodemográficas y clínicas se muestran en la tabla 1 y 2 respectivamente.

En cuanto a las características generales de la muestra, el número de mujeres ha sido muy superior al de varones (un $73 \%$ y un $27 \%$, respectivamente). Los participantes tenían una media de edad de 50 años (SD=13,3 años, rango 18-90 años) y la mayoría estaban casados o con pareja estable (73\%). El 66\% de los usuarios era paciente y el $34 \%$ restante familiar. En el grupo de familiares los grados de parentesco más 
Tabla 1. Datos sociodemográficos de la muestra.

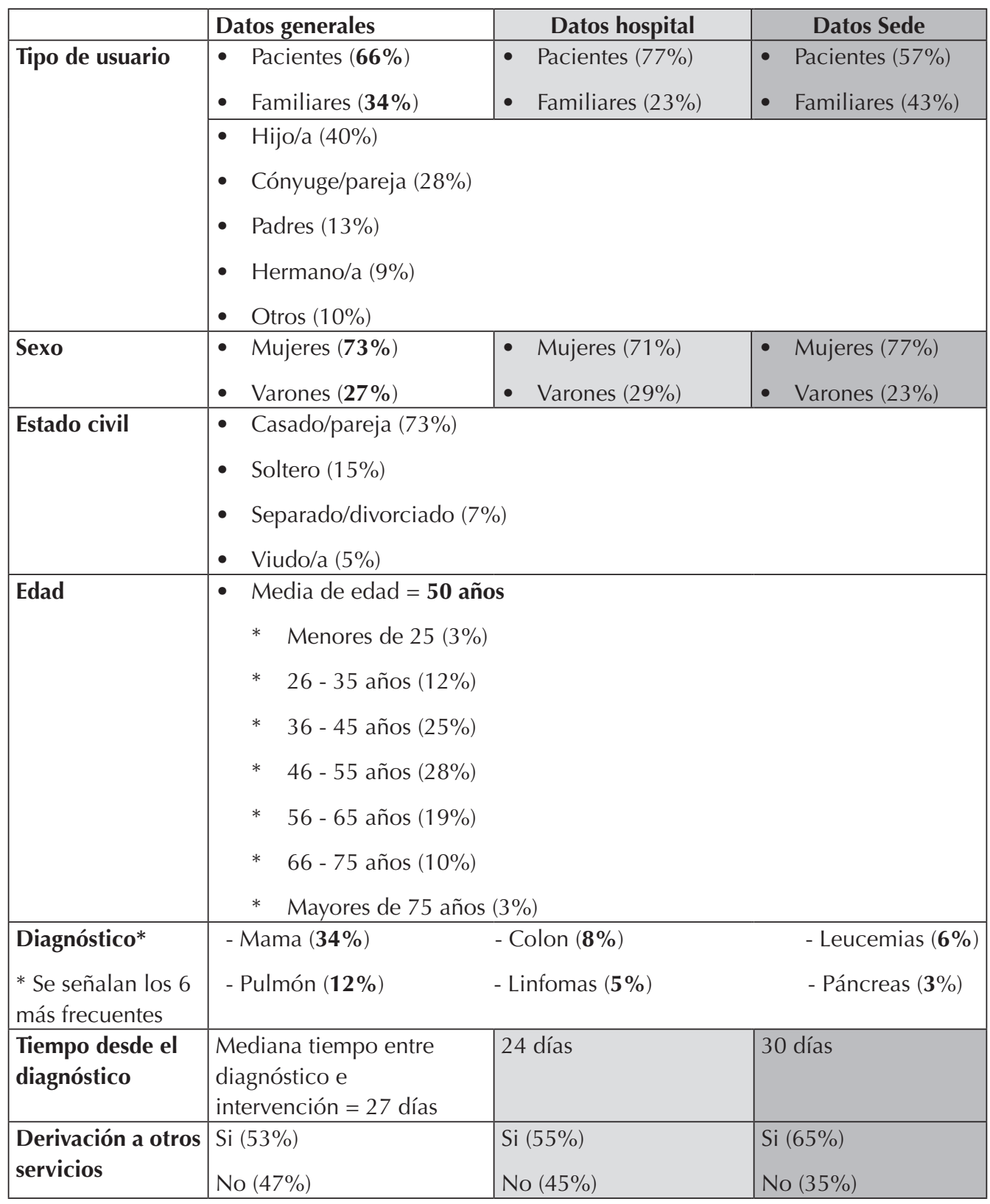

representados fueron hijo/a (40\%), cónyuge/pareja (28\%) y padre/madre (13\%). Los diagnósticos oncológicos más frecuentes fueron cáncer de mama (34\%), cáncer de pulmón $(12 \%)$, cáncer de colon $(8 \%)$, leucemias $(6 \%)$ y linfomas (5\%). La mediana de tiempo entre el diagnóstico de cáncer y la intervención fue de 27 días ( $S D=24$ días, rango 0-90 días), encontrándose un $58 \%$ de casos en el intervalo correspondiente a menos de 30 días frente al $42 \%$ restante que se distribuyen en el intervalo 
Tabla 2. Datos clínicos de la muestra.

\begin{tabular}{|c|c|c|c|c|c|}
\hline & \multicolumn{2}{|l|}{ Datos generales } & \multicolumn{2}{|c|}{ Datos hospital } & Datos Sede \\
\hline \multirow{13}{*}{$\begin{array}{l}\text { Grado de } \\
\text { malestar } \\
\text { media distrés } \\
\text { del total de } \\
\text { usuarios } 5,4\end{array}$} & \multicolumn{2}{|c|}{$\begin{array}{l}\text { - Sin malestar o poco significativo } \\
(10 \% \text { de los usuarios })\end{array}$} & \multicolumn{2}{|r|}{$(8 \%)$} & $(12 \%)$ \\
\hline & \multicolumn{2}{|l|}{ - $\quad$ Malestar mínimo (15\%) } & & $(19 \%)$ & $(12 \%)$ \\
\hline & \multicolumn{2}{|l|}{ - $\quad$ Malestar moderado (20\%) } & & $(23 \%)$ & $(15 \%)$ \\
\hline & \multicolumn{2}{|l|}{ - $\quad$ Malestar alto $(31 \%)$} & & $(30 \%)$ & $(31 \%)$ \\
\hline & \multicolumn{2}{|l|}{ - $\quad$ Malestar severo $(25 \%)$} & & $(20 \%)$ & $(31 \%)$ \\
\hline & \multirow{2}{*}{$\begin{array}{l}\text { Media distrés pacientes }=5,2 \\
\text { Media distrés familiares }=5,9 \\
\text { Media distrés padres }=7,2\end{array}$} & \multirow[b]{2}{*}{ Paciente } & \multicolumn{2}{|c|}{ Familiar } & \multirow[b]{2}{*}{ Padres } \\
\hline & & & total & $\begin{array}{c}\sin \\
\text { padres }\end{array}$ & \\
\hline & $\begin{array}{lll} & \text { Sin malestar o poco } \\
\text { significativo } & & \\
\end{array}$ & $10 \%$ & $9 \%$ & $7 \%$ & $2 \%$ \\
\hline & - Malestar mínimo & $17 \%$ & $9 \%$ & $11 \%$ & $0 \%$ \\
\hline & - $\quad$ Malestar moderado & $21 \%$ & $19 \%$ & $20 \%$ & $15 \%$ \\
\hline & - Malestar alto & $31 \%$ & $32 \%$ & $32 \%$ & $36 \%$ \\
\hline & - $\quad$ Malestar severo & $21 \%$ & $46 \%$ & $30 \%$ & $47 \%$ \\
\hline & \multicolumn{5}{|c|}{$\begin{array}{l}\text { Mayor proporción de distrés alto/severo en familiares que en pacientes } \\
(78 \% \text { versus } 52 \% \text { ) debido a la mayor proporción de dichos niveles en los } \\
\text { padres de menores de } 25 \text { años ( } 83 \%)\end{array}$} \\
\hline \multirow{5}{*}{$\begin{array}{l}\text { Necesidades } \\
\text { detectadas }\end{array}$} & \multicolumn{4}{|l|}{ No necesidad } & $36 \%$ \\
\hline & \multicolumn{4}{|l|}{ Si necesidad } & $64 \%$ \\
\hline & \multicolumn{5}{|c|}{ Necesidad emocional (75\%) } \\
\hline & \multicolumn{5}{|c|}{ Necesidad social (15\%) } \\
\hline & \multicolumn{5}{|c|}{ Necesidad médico-sanitaria (10\%) } \\
\hline
\end{tabular}

correspondiente a más de 31 días. En lo referente al lugar donde se desarrollar la intervención, el $51 \%$ de los pacientes y familiares fueron vistos en hospital frente al $49 \%$ que recibieron la intervención en la sede de la AECC (ver tabla 1).

Respecto a las características clínicas referidas a niveles de distrés, la media en la puntuación del TD del total de usuarios fue de 5,4, lo que significa un nivel de distrés moderado. La distribución de los pacientes y familiares en los puntos que conforman el TD fueron los siguientes; sin malestar o poco significativo $(10 \%)$, malestar mínimo $(15 \%)$, malestar moderado $(20 \%)$, alto $(31 \%)$ y malestar severo $(25 \%)$. Lo que implica que un $56 \%$ de beneficiarios presentaron niveles de distrés altos o severos. Si bien, encontramos datos claramente diferenciados cuando los niveles de distrés se analizan en función del lugar en el que se había desarrollado la intervención (hospital/sede AECC) y en función de si el usuario era paciente o familiar (ver ta- 
bla 2). En relación a las necesidades detectadas, la proporción de sujetos que presentan necesidad es superior a aquellos que no manifiestan necesidad ( $64 \%$ frente al $36 \%$ ). Entre aquellos con necesidad específica, un $75 \%$ presentan necesidad emocional, un $15 \%$ social y un $10 \%$ necesidad medico-sanitaria. Hasta el $54,5 \%$ de los casos necesitan derivación a un servicio especializado de atención, principalmente el servicio de atención psicológica.

Respecto a la posible asociación entre las diferentes variables recogidas y el nivel de distrés, la media distrés en mujeres es ligeramente superior a los varones (5,52 y 5,22 respectivamente). En general se ob- serva que las mujeres se sitúan en mayor medida que los varones en los intervalos superiores de distrés, pero estas diferencias no alcanzan la significación estadística $\left(\chi^{2}=0,506 ; p=0,477\right)$. En esta misma línea, el estado civil tampoco mostró asociación con el nivel de distrés. Por el contrario, si mostraron una asociación significativa las siguientes variables: tipo de beneficiario, esto es, si es enfermo o familiar $\left(\chi^{2}=11,29 ; p=0,001\right)$, la localización del tumor $\left(\chi^{2}=44,37 ; p=0,000\right)$, los días transcurridos desde el diagnóstico $(\leq 30$ o $>30)$ $\left(\chi^{2}=8,008 ; p=0,005\right)$ y la edad de la persona evaluada $(<35 ; 36-55 ; 56-65 ;>65)$ $\left(\chi^{2}=28,617 ; p=0,000\right)$. Así, los familiares

\section{Tabla 3. Análisis univariado y multivariado de los factores de riesgo de distrés alto y severo.}

\begin{tabular}{|c|c|c|c|c|}
\hline Variable & Wald & B & E.T. B & OR \\
\hline Tipo beneficiario & $0,19^{*}$ & $-0,76$ & 0,17 & 0,93 \\
\hline Edad & $21,91^{*}$ & & & \\
\hline 36 a 55 años & & 0,98 & 0,28 & 2,68 \\
\hline 56 a 65 años & & 0,78 & 0,20 & 2,19 \\
\hline Más 65 años & & 1,01 & 0,24 & 2,75 \\
\hline Días transcurridos & $1,80^{*}$ & 0,19 & 0,14 & 1,21 \\
\hline Localización Tumoral & $38,25^{*}$ & & & \\
\hline Mama & & $-0,53$ & 0,47 & 0,59 \\
\hline Colorrectal & & 0,64 & 0,52 & 1,91 \\
\hline Pulmón & & 0,23 & 0,49 & 1,26 \\
\hline Linfoma & & 0,07 & 0,54 & 1,07 \\
\hline Leucemia & & 1,23 & 0,66 & 3,43 \\
\hline Ginecológico & & 0,06 & 0,56 & 1,06 \\
\hline Páncreas & & 0,23 & 0,57 & 0,68 \\
\hline Estómago & & $-0,38$ & 0,57 & 0,68 \\
\hline Hígado & & 0,12 & 0,63 & 1,13 \\
\hline
\end{tabular}


presentan mayores niveles de distrés que los pacientes, especialmente los padres. A medida que aumenta el número de días trascurridos desde el diagnostico el nivel de distrés disminuye. Los sujetos más jóvenes muestran niveles de distrés superiores a los de mayor edad.

Con el objeto de explorar posibles predictores asociados al distrés en pacientes y familiares recién diagnosticados se incluyeron las variables que habría mostrado significación estadística a un análisis multivariante de regresión logística. El modelo final obtenido posee una sensibilidad del $96,3 \%$ y una especificidad del $11,6 \%$, clasificando correctamente al 69,3\% del total de sujetos. Finalmente, explica el $12,3 \%$ de la varianza del distrés. Se compuso de las siguientes variables: tipo de beneficiario (enfermo o familiar), edad de la persona evaluada $(<35 ; 36-55 ; 56-65 ;>65)$, días transcurridos desde el diagnóstico $(\leq 30$ o >30) y localización tumoral (ver tabla 3).

Por último, señalar las diferencias encontradas en función del profesional que lleva a cabo la intervención (médico, trabajador social, psicólogo y coordinador de voluntariado) en cuanto a nivel de distrés y necesidades detectadas. El 26\% de los casos evaluados por los psicólogos/as presentaban un nivel leve de distrés frente a un $74 \%$ con un nivel moderado-severo. En el caso de otros profesionales el 43\% de los beneficiarios presentan distrés leve, mientras un $57 \%$ presenta un nivel moderado-severo. Respecto a detección de necesidad, esta se detectó en el 70,4\% de los casos evaluados por psicólogos frente a un $54 \%$ de los casos evaluados por otros profesionales. Se observan diferencias significativas tanto en el nivel de distrés $\left(\chi^{2}=43,19 ; p=0,000\right)$ como en la necesidad detectada $\left(\chi^{2}=40,14 ; p=0,000\right)$ en función de si el evaluador es psicólogo u otro profesional.

\section{DESAFÍOS Y PERSPECTIVAS DE FUTURO}

En las últimas décadas, ha ido in crescendo el cuerpo de literatura científica que ha documentado la necesidad de desarrollar en los servicios de oncología programas de detección de distrés dirigidos a pacientes oncológicos y sus familiares. Asimismo, su aplicación clínica es contundente y está fundamentada por numerosos estudios $^{(27-30)}$ que han puesto de manifiesto los beneficios derivados de los mismos en términos de mejoras en calidad de vida, adhesión a recomendaciones terapéuticas y mayor satisfacción con la atención recibida $^{(31-33)}$. A pesar de ello, son muchos los aspectos que permanecen, aún, sin aclarar acerca de los aspectos más prácticos para su adecuada ejecución e implantación.

\section{Dificultades en la ejecución del programa en la práctica clínica diaria en los hospitales}

Como en otro tipo de intervenciones que se realizan en el ámbito hospitalario, conseguir una implantación generalizada depende de muchos actores: políticos, gestores, profesionales sanitarios y los pacientes. Por parte de los pacientes, no parece existir problemas para la implantación del programa, dada la alta aceptación del mismo. A pesar de ello, aún se podría mejorar la tasa de cobertura si generamos las estrategias oportunas para llegar a aquellos pacientes y familiares que a pesar de haber sido informados sobre el programa consideran que no es el momento de acudir, que lo harán más adelante y que finalmente se olvidan de hacerlo o de aquellos que a pesar de experimentar problemas significativos que producen un elevado nivel de distrés declinan la intervención, tal vez en favor del apoyo informal de su familia y amigos.

Respecto a los profesionales sanitarios de los diferentes servicios implicados, mayoritariamente, encuentran distintas moti- 
vaciones para informar a pacientes y familiares sobre el programa, que van desde el beneficio que supone para sus pacientes hasta la propia satisfacción personal del trabajo en equipo. Sin embargo, la realidad se encuentra con barreras no siempre fáciles de solventar; la escasez de tiempo en consulta y el olvido a la hora de ofrecer la información a los pacientes son las más citadas por los profesionales, si bien no son las únicas. En este sentido, el principal hándicap con el que nos hemos encontrado ha sido que algunos de los profesionales sanitarios solo ofrecían información del programa a los pacientes que ya presentaban un importante nivel de distrés. Sin duda, los equipos deben tratar de ofrecer la información sobre los servicios de ayuda de forma que todos los pacientes puedan beneficiarse de los mismos. Actualmente, aún necesitamos identificar con mayor detalle los aspectos prácticos en la ejecución del programa en la práctica clínica diaria, pero ya estamos en disposición de ofrecer algunos aspectos que determinan el óptimo desarrollo del programa en el hospital. Así, es necesario un apoyo institucional claro, explícito y público de las administraciones sanitarias y una implicación por parte de los profesionales que garantice que la información sobre el programa llegue a los usuarios. Para conseguirlo, las estrategias que se han mostrado más eficaces han sido: presentación en las distintas áreas clínicas; establecimiento de acuerdos con determinados servicios para incorporación funcional; identificación, formación y entrenamiento de informantes clave y distribución de material de difusión.

\section{Dificultades para determinar el flujo de derivación óptimo}

Uno de los problemas más citados para la puesta en marcha de un protocolo de detección de distrés en pacientes de cáncer es la preocupación por el hecho de que una elevada detección de pacientes con niveles de distrés significativos puede sobrecargar los servicios psicosociales. Sin embargo, los datos no apoyan esta afirmación. Así, el estudio llevado a cabo por Carlson y colaboradores entre más de 1.100 pacientes con cáncer de mama y pulmón destaca que sólo entre 40\% y 50\% de los pacientes aceptó una consulta telefónica y solo el $30 \%$ acude finalmente al servicio $^{(34)}$. En la misma línea, en el trabajo de Verdonck realizado con pacientes con cáncer de cabeza y cuello señala que únicamente el $20 \%$ de los pacientes con niveles de distrés Ilegaron al servicio de derivación ${ }^{(35)}$ y el grupo de Shimizu observó que de aquellos pacientes que mostraron elevados niveles de sufrimiento, sólo el $25 \%$ optó por acudir al servicio al que fue derivado ${ }^{(36)}$.

Nuestra experiencia en este sentido, es claramente diferente, un $70 \%$ de los usuarios con niveles de distrés significativos que fueron derivados Ilegan al servicio de derivación. El porcentaje de usuarios perdidos viene determinado por el lugar donde se realiza la intervención, así varía entre un $11 \%$ cuando se realiza en formato presencial en las sedes de la AECC, un $23 \%$ por vía telefónica y un $40 \%$ cuando se desarrolla en un centro hospitalario. El perfil de los usuarios que han participado en el protocolo pero no llegan al servicio de derivación es el de varones y mayores de 65 años.

\section{Elevados niveles de distrés y diferencias en la detección en función del perfil del profesional que desarrolla la evaluación}

En líneas generales, el nivel de distrés significativo que hemos encontrado en la población atendida es superior al nivel documentado por otros autores y grupos de investigación. Concretamente en nuestro programa el $75 \%$ de los sujetos informó la presencia de distrés significativo, con 
un punto de corte de 4 o más en el DT, lo cual es claramente superior a lo descrito por autores como Almanza $(61,8 \%)^{(37)}$, Zabora $(31 \%)^{(38)}$, Pascoe $(35 \%)^{(39)}$, Dolbeaut $(38 \%)^{(40)}$, Trask $(50 \%)^{(41)}$, Ozalp $(59 \%)^{(42)}$ y Graves $(61,6 \%)^{(43)}$. Discrepancias que probablemente obedecen a diferencias metodológicas, trans-culturales (Australia, Estados Unidos de América, Francia, México, etc.), y a los distintos momentos de evaluación de los correspondientes estudios. Respecto a las diferencias metodológicas destacar dos de gran relevancia. La primera de ellas hace referencia a la población objetivo. Nuestros datos incluyen tanto a pacientes como familiares, aspecto que no concurre en el resto de los estudios citados que se circunscriben a pacientes. Este hecho es de interés a la hora de explicar que nuestra población muestre un nivel superior de distrés, dado que los familiares, principalmente el subgrupo de padres, informan de distrés más elevado que los pacientes. Otro aspecto diferencial es el momento de medida, en nuestro caso la detección de distrés se produce en momentos muy próximos al diagnostico.

Quizá el hecho que más ha Ilamado nuestra atención se refiere a las diferencias que hemos encontrado en la detección de distrés significativo en función del perfil del profesional que realiza la evaluación. Así, cuando el protocolo de detección es realizado por un psicólogo se producen una mayor tasa de casos positivos, entendidos éstos como distrés significativo y presencia de necesidad, que cuando es realizado por otro perfil profesional. En principio, este hallazgo sería coincidente con la literatura que señala que los profesionales de la salud no psicólogos tienen más dificultad para reconocer el distrés en los pacientes ${ }^{(13,44)}$. De ser así, la estrategia sería relativamente fácil, únicamente tendríamos que reforzar la formación que se realiza con estos profesionales para facilitar su labor de detección. Sin embar- go, el hecho de que los niveles de distrés de los profesionales no psicólogos tengan mayor similitud con los publicados y a la magnitud de las diferencias entre los niveles detectados por unos profesionales y otros nos hace sospechar que hay más variables implicadas. Variables, que merece un mayor nivel de análisis futuro que nos permita discernir sobre las siguientes cuestiones ¿los profesionales no psicólogos tienen más dificultades para detectar el distrés? o, por el contrario, ¿por qué los psicólogos detectan más casos con distrés significativo?, ¿será que cuando el profesional no psicólogo desarrolla el protocolo funciona cómo un programa de screening y cuándo lo desarrolla un psicólogo funciona como un programa de evaluación y atención?

\section{REFERENCIAS BIBLIOGRÁFICAS}

1. Bayés R. Una estrategia para la detección del sufrimiento en la práctica clínica. Rev Soc Esp Dolor 2000;7:70-4.

2. Derogatis LR, Morrow GR. The prevalence of psychiatric disorders among cancer patients. JAMA 1983;249:751-7.

3. Massie MJ, Holland JC. Depression and the cancer patient. J Clin Psychiatry 1990; 51 Suppl 12-7, discussion,1990;18-9.

4. Hopwood P, Stephens RJ. Depression in patients with lung cancer prevalence and risk factors derived from quality o life data. J Clin Oncol 2000;18:893-903

5. Grassi L, Travado L, Gil F, Sabato S, Rossi E; and de SEPOS Group. Psychosocial morbidity and its correlatos in cancer patients of the Mediterranean area: findings from the Southern European Psycho-Oncology Study. J Affect Disord 2004;83:243-8.

6. Gil F, Costa G, Pérez FJ, Salamero M, Sánchez N, Sirgo A. Adaptación psicológica y prevalencia de transtornos mentales en pacientes con cáncer. Med Clin (Barc) 2008;130(3):90-2.

7. Arranz P, Ulla S, Coca C. Leucemias, linfo- 
mas y mielomas. En Die Trill M. Psicooncología. Madrid: ADES; 2003.

8. Iscoe $\mathrm{N}$, Williams $\mathrm{Jl}$. Prediction of psychosocial distress in patients with cancer. In: Osoba D, editor. Effect of cancer on quality of life, CRC Press: Boca RatonBoston-Ann Arbor-London, 1991. p. 41-59.

9. Vargas G, Espósito G. Dolor y cuidados Paliativos en Oncología: evaluación, manejo, y tratamiento. Caracas, Venezuela: Editorial Expansión Científica; 1999.

10. Fallowfield LJ, Hall A. Psychosocial effects of being offered choice of surgery for breast cancer. BMJ 1994; 309-448.

11. Kiss A, Cavalli F, Cull A, Gallmeier WM, Hürny $C$, Keller $M$, et al. Psychosocial/ psychotherapeutic interventions in cancer patients: consensus statement. Supp Care Cancer 1995;3:270-1.

12. National Comprehensive Cancer Network. Practice Guidelines in Oncology, Version 1; 2002: National Comprehensive Cancer Network. Distress Management. Fort Washington, PA; 2002.

13. Carlson LE, Waller A, Mitchell, AJ. Screening for distress and unmet needs in patients with cancer: review and recommendations. J Clin Oncol 2012;30(11):1160-77.

14. Söllner. W, DeVries. A, Steixner E, Lukas P, Sprinzl G, Rumpold G, Maislinger S. How successful are oncologists in identifying patient distress, perceived social support, and need for psychosocial counselling? Br J Cancer 2001; 84(2). 179-85. Doi: 10.1054/bjoc.2000.1545

15. Mitchell JT. Essencial factors for Effective Psychological response to disasters and other crises. Int J Emerg Ment Health, 1999;19:299-325.

16. Hutchison SD, Steginga SK, Duna J. The tiered model of psychosocial intervention in cancer: A community based approach. Psychooncology 2006;15(6):541-6. Doi: 10.1002/pon.973

17. Heinonen $\mathrm{H}$, Volin L Uutela A, Zevon $\mathrm{M}$, Barrick C, Ruutu T. Quality of life and factors related to perceived satisfaction with quality of life after allogeneic bone marrow transplantation. Ann Hematol 2001;0:13743. Doi: 10.1007/s002770000249

18. Kissane DW, McKenzie M, McKenzie DP, Forbes A, O'Neill I, Bloch S. Psychosocial morbidity associated with patterns of family functioning in palliative care: baseline data from the Family Focused Grief Therapy controlled trial. Palliat Med 2003; 17 (6): 527-37. Doi:10.1191/0269216303pm808oa

19. Zabora JR. Screening procedures for psychosocial distress. En: Holland JC, Breitbart W, Jacobsen PB, et al., editors. Psycho-oncology. New York, NY: Oxford University Press; 1998. p. 653-61.

20. Hoffman BM, Zevon MA, D'Arrigo MC, Cecchini TB. Screening for distress in cancer patients: the NCCN rapid-screening measure. Psychooncology 2004; 13 (11): 792-9. Doi: 10.1002/pon.796

21. Hegel MT, Collins ED, Kearing S, Gillock KL, Moore CP, Ahles TA. Sensitivity and specificity of the Distress Thermometer for depression in newly diagnosed breast cancer patients. Psychooncology 2008;17(6):55660. Doi: 10.1002/pon.1289

22. Akizuki N, Akechi T, Nakanishi T, Yoshikawa E, Okamura M, Nakano T, et al. Development of a brief screening interview for adjustment disorders and major depression in patients with cancer. Cancer 2003;97:2605-13. Doi: 10.1002/ cncr. 11358

23. Jacobsen PB, Donovan KA, Trask PC, Fleishman SB, Zabora J, Baker F, et al. Screening for psychologic distress in ambulatory cancer patients: a multicenter evaluation of the Distress Thermometer. Cancer 2005;103:1494-502. Doi: 10.1002/ cncr.20940

24. Hawkes A, Hughes K, Hutchison S, et al. Feasibility of brief psychological distress screening by a community-based telephone helpline for cancer patients and carers. BMC Cancer 2010;10:14.

25. Campbell A, Steginga $S$, Ferguson $M$, et 
al. Measuring distress in cancer patients: The distress thermometer in an Australian sample. Prog Palliat Care, 2009; 17:61-68. Doi:10.1179/096992609X392259

26. Gessler S, Low J, Daniells E, et al. Screening for distress in cancer patients: Is the Distress Thermometer a valid measure in the UK and does it measure change over time? A prospective validation study. Psychooncology 2008; 17:538-47. Doi:10.1002/pon.1273

27. Carroll BT, Kathol R, Noyes R, et al. Screening for depression and anxiety in cancer patients using the hospital anxiety and depression scale. Gen Hosp Psychiatry 1993; 15: 69-74. Doi:10.1016/01638343(93)90099-A

28. Holland JC, Anderson B, et al. NCCN Guidelines for Distress Management. JNCCN 2007; 5:66-98.

29. Pascoe S, Edelman S, Kidman A. Prevalence of psychological distress and use of support services by cancer patients at Sydney Hospitals. Aust N Zeal J Psychiatr 2001;34:785-91.

30. Ozalp E, Cankurtaran ES, Soygür H, Geyik PO, Jacobsen PB. Screening for psychological distress in Turkish cancer patients. Psychooncology 2007; 16(4): 304-11. Doi:10.1002/pon.1059

31. Shim EJ, Mehnert A, Koyama A, et al. Health-related quality of life in breast cancer: A cross-cultural survey of German, Japanese, and South Korean patients. Breast Cancer Res Treat, 2006;99:341-50.

32. Von Essen L, Larsson G, Oberg K, Sjödén PO. "Satisfaction with care": Associations with health related quality of life and psychosocial function among Swedish patients with endocrine gastrointestinal tumours. Eur J Cancer Care (Engl), 2002; 11:91-99. Doi: 10.1046/j.1365-2354.2002.00293.x

33. Hamer M, Chida Y, Molloy GJ. Psychological distress and cancer mortality. J Psychosom Res 2009;66:255-258. Doi:10.1016/j. jpsychores.2008.11.002

34. Carlson LE, Groff SL, Maciejewski O,
Bultz BD. Screening for distress in lung and breast cancer outpatients: A randomized controlled trial. J Clin Oncol, 2010; 28:4884-91.

35. Verdonck-de Leeuw IM, de Bree R, Keizer AL, Houffelaar T, Cuijpers P, Mecheline $H$. van der Linden, et al. Computerized prospective screening for high levels of emotional distress in head and neck cancer patients and referral rate to psychosocial care. Oral Oncol 2009; 45:e129-e133. Doi: 10.1016/j.oraloncology.2009.01.012

36. Shimizu K, Ishibashi Y, Umezawa S, Izumi H, Akizuki N, Ogawa A et al. Feasibility and usefulness of the "Distress Screening Program in Ambulatory Care" in clinical oncology practice. Psychooncology 2010; 19:718-25. Doi: 10.1002/pon.1616

37. Almanza MJJ, Rosario JI, Pérez S. Traducción, adaptación y validación del Termómetro de Distrés en una muestra de pacientes mexicanos con cáncer. Rev Sanid Milit Mex 2008; 62(5):209-17.

38. Zabora J, Brintzenhofeszoc K, Curbow B, Hooker C, Piantadosi S.The prevalence of distress by cancer site. Psychooncology 2001; 10: 19-28. Doi: .1002/1099-1611(200101/02)10:1<19::AIDPON501>3.0.CO;2-6

39. Pascoe S, Edelman S, Kidman A. Prevalence of psychological distress and use of support services by cancer patients at Sydney Hospitals. Aust N Zeal J Psychiatr 2001;34:785-91. Doi: 10.1046/j.14401614.2000.00817.x

40. Dolbeault S, Bredart A, Mignot V, Hardy $P$, et al. Screening for psychological distress in two French cancer centers: feasibility and performance of the adapted distress thermometer. Palliat Support Care 2008;6(2):107-17. Doi: 10.1017/ S1478951508000187

41. Trask PC, Paterson A, Riba M, Brines B, Grifith K, Parker P, et al. Assessment of psychological dsitress in prospective bone marrow transplant patients. Bone Ma- 
rrow Transplant 2002;29(11):917-25. Doi: 10.1038/sj.bmt.1703557

42. Ozalp E, Cankurtaran ES, Soygür H, Geyik PO, Jacobsen PB. Screening for psychological distress in Turkish cancer patients. Psychooncology 2007;16(4): 304-11. Doi:10.1002/pon.1059

43. Graves KD, Arnold SM, Love CL, Kirsh $\mathrm{KL}$, Moore PG, Passik SD. Distress screening in a multidisciplinary lung cancer clinic: prevalence and predictors of clinically significant distress. Lung Cancer 2007; 55(2): 215-24. Doi: 10.1016/j. lungcan.2006.10.001

44. Mitchell A, Vahabzadeh A, Magruder K. Screening for distress and depression in cancer settings: 10 lessons from 40 years of primary-care research. Psychooncology 2011; 20:572-84. Doi:10.1002/pon.1943 